\title{
Impacto do uso de informações demográficas para a recomendação de aplicativos móveis
}

\author{
Raissa P. P. M. Souza ${ }^{1}$, Leonardo J. A. dos $\operatorname{Santos}^{1}$, Mateus P. Silva ${ }^{1}$, \\ Fabrício A. Silva ${ }^{1}$, Thais R. M. B. Silva ${ }^{1}$ \\ ${ }^{1}$ Núcleo de Estudos em Sistemas Pervasivos e Distribuídos \\ Instituto de Ciências Exatas e Tecnológicas \\ Universidade Federal de Viçosa (UFV) - Florestal - MG - Brasil \\ \{raissa.polyanna, leonardo.j.alves, mateus.p.silva, \\ fabricio.asilva, thais.braga\}eufv.br
}

\begin{abstract}
Recently, we are facing a significative increase in the number of mobile devices and applications. Given the large number of available applications, users have difficult to select the ones to install. On the other hand, applications' providers have difficult in selecting users to target their advertisements in order to increase the convertion rate. In this work, we propose a solution to recommend applications based on the similarities of users and applications, as well as on the demographic information of the users' region of residence. We evaluate our proposal on real data from 7,406 users, and the results reveal an improvement up to 14 percentage points when comparing to a traditional solution that does not rely on the demographic information. In addition, the potential conversion rate increases by up to 30 times when compared to samples of users taken randomly.
\end{abstract}

Resumo. Nos últimos anos, tem-se percebido um aumento significativo no uso de dispositivos móveis e aplicativos. Considerando o grande número de aplicativos, usuários enfrentam dificuldades em decidir qual instalar. Por outro lado, as provedoras de aplicativos enfrentam dificuldades para selecionar usuários para direcionar campanhas de marketing com o objetivo de aumentar a taxa de conversão. Neste trabalho, é proposta uma solução que faz a recomendação de aplicativos com base em dados de similaridades entre usuários e entre aplicativos, e também em informações demográficas da região de residência do usuário. Foi feita uma avaliação com dados reais de 7.406 usuários, e os resultados mostraram que a solução proposta se sobressaiu em até 14 pontos percentuais de precisão quando comparada com uma solução tradicional que não considera os dados demográficos. Além disso, a potencial taxa de conversão aumentou em até 30 vezes, quando comparada a usuários selecionados aleatoriamente.

\section{Introdução}

Nos últimos anos, tem-se percebido um crescimento significativo no uso de dispositivos móveis e seus respectivos aplicativos [Intelligence 2019]. Com um número cada vez maior de aplicativos nas lojas digitais, está cada vez mais difícil para o usuário escolher qual o aplicativo de determinado segmento poderá atender às suas necessidades. Por outro 
lado, as provedoras de aplicativos têm dificuldade em escolher usuários-alvo para direcionarem suas propagandas, visando aumentar o retorno sobre o investimento em marketing, em termos da taxa de conversão (i.e., total de usuários que instalaram o aplicativo dentre os que foram alvo de alguma campanha).

Uma estratégia para mitigar esse problema visa aplicar algoritmos de recomendação com base em similaridades entre os usuários em termos de seus aplicativos instalados. A ideia por trás dessa estratégia é que usuários que possuem instalados alguns aplicativos em comum, possuem interesses e características em comum que podem levar à instalação de outros aplicativos. Por exemplo, se um usuário possui instalado iFood e Rappi, e é conhecido que a maioria dos usuários que possuem esses dois aplicativos instalados também possuem o Uber instalado, a recomendação desse último aplicativo para o usuário pode ter uma boa chance de ser assertiva.

Apesar de intuitivo, a premissa de que apenas a similaridade em termos dos aplicativos instalados é relevante pode ser considerada fraca. A instalação de algum aplicativo leva em consideração vários outros aspectos além dos técnicos. Por exemplo, o Uber pode não estar disponível na localidade de residência do usuário. Ou seja, apenas saber que o usuário possui iFood e Rappi não é um indicativo forte para a instalação do Uber. Além disso, alguns aplicativos podem ser mais atrativos para alguma faixa etária ou de renda. Com isso em mente, a hipótese deste artigo é que é possível melhorar a qualidade de recomendações de aplicativos, se forem consideradas também as informações demográficas da região em que o usuário reside (e.g., distribuição de renda, idade, gênero, nível de alfabetização, dentre outros).

O objetivo deste trabalho é validar essa hipótese. Para isso, foram implementadas duas soluções de recomendação. A primeira é baseada apenas nas similaridades entre os usuários em termos dos aplicativos instalados, nomeada como ALBERTA (AppLication BasEd RecommendaTion Algorithm). A segunda utiliza, além da similaridade em termos de aplicativos, a similaridade em termos das características da região de residência dos usuários, chamada de ANCESTOR (Application aNd CEnsus baSed recommendaTion algORithm). Foram utilizados dados de 249 aplicativos instalados de 7.406 usuários reais, coletados durante dois meses. Os resultados mostraram que, quando as informações demográficas são consideradas, a precisão melhora em até 14\% em alguns casos.

Este texto está organizado da seguinte forma. Na Seção 2 são apresentados os principais estudos relacionados. Em seguida, na Seção 3 os dados utilizados no trabalho são apresentados. A solução proposta e os resultados alcançados são apresentados na Seção 4. Por fim, as conclusões e trabalhos futuros são discutidos na Seção 5.

\section{Trabalhos Relacionados}

Os trabalhos [Frey et al. 2017, Cheng et al. 2018] utilizam LDA (Latent Dirichlet Allocation) para fazer as recomendações. O primeiro utilizou LDA para selecionar os tópicos principais dentre as descrições de aplicativos, utilizando a probabilidade de o usuário gostar de cada tópico como insumo para um modelo baseado no algoritmo Floresta Aleatória. Já os autores do segundo trabalho utilizam a ordem de instalação dos aplicativos para observar aspectos do uso dos aplicativos.

Outros dois trabalhos utilizam grafos para representar associações entre usuários. No trabalho de [Pan et al. 2011] são utilizadas informações de sensores de smartphones 
Tabela 1. Categorização dos Trabalhos Relacionados

\begin{tabular}{|l|c|c|l|}
\hline \multicolumn{1}{|c|}{ Trabalho } & $\begin{array}{c}\text { Filtro } \\
\text { Colaborativo }\end{array}$ & $\begin{array}{c}\text { Outras } \\
\text { Técnicas }\end{array}$ & \multicolumn{1}{c|}{ Detalhes } \\
\hline [Frey et al. 2017] & & $\mathrm{X}$ & LDA e Floresta Aleatória \\
\hline [Cheng et al. 2018] & & $\mathrm{X}$ & LDA \\
\hline [Pan et al. 2011] & & $\mathrm{X}$ & Grafo \\
\hline [Xu et al. 2018] & & $\mathrm{X}$ & Grafo e PageRank \\
\hline [Ma et al. 2016] & & $\mathrm{X}$ & Modificação de Word2 Vec \\
\hline [Yin et al. 2017] & $\mathrm{X}$ & $\mathrm{X}$ & Bag of Words e Tópicos Latentes \\
\hline [Peng et al. 2018] & $\mathrm{X}$ & & Matriz de Fatorização \\
\hline [Liu et al. 2015] & $\mathrm{X}$ & & Fatores Latentes \\
\hline [Liu et al. 2016] & $\mathrm{X}$ & & Fatores Latentes \\
\hline Trabalho Atual & $\mathrm{X}$ & & Estratégia Item-Item \\
\hline
\end{tabular}

para construir um grafo que representa ligações entre usuários, possibilitando o cálculo do potencial de instalação de um aplicativo com base nos vizinhos de um determinado usuário. Já o trabalho elaborado por [Xu et al. 2018] considera a funcionalidade de cada aplicativo, fazendo com que seja possível predizer quais as próximas necessidades do usuário através de um grafo de co-ocorrência.

O trabalho desenvolvido por [Ma et al. 2016] realiza uma alteração no algoritmo Word2Vec, visando predizer a instalação de aplicativos com base na utilização recente de outros. Já o trabalho desenvolvido por [Yin et al. 2017] utiliza preferências de permissões para indicar aplicativos, por meio da descrição e permissões de cada aplicativo.

Outros trabalhos levam em consideração as preferências de privacidade dos usuários. O trabalho de [Peng et al. 2018] identifica os aplicativos que requisitam muitas permissões e possuem classificação inferior, dando a eles baixa prioridade. Da mesma forma, o trabalho de [Liu et al. 2015] também relaciona as preferências de privacidade e de comportamento do usuário. Entretanto, estes fatores são relacionados em um perfil latente. Posteriormente, os autores modificaram este trabalho adotando uma nova estratégia, onde é analisada a categoria e funcionalidade de cada aplicativo [Liu et al. 2016]. Mais uma vez, são utilizados vetores latentes, agora relacionando as funcionalidades de cada aplicativo aos interesses do usuário através da lista de instalações deste.

A Tabela 1 apresenta uma comparação entre os trabalhos existentes. Entre os estudos citados acima, é comum encontrar soluções baseadas em uma das vertentes do Filtro Colaborativo. Porém, a maioria dos trabalhos requer dados que são mais custosos de se conseguir. Em contrapartida, a solução proposta neste artigo utiliza somente uma captura dos aplicativos instalados por um usuário e sua localização aproximada. Dessa forma, é criado um modelo estocástico, onde acredita-se que os aplicativos instalados no atual momento do tempo, em conjunto com informações demográficas da sua região de residência, refletirão nos aplicativos que tendem a ser instalados no futuro.

\section{Descrição dos Dados}

Para realizar o estudo proposto neste trabalho, foi utilizado um conjunto de dados reais de 7.406 usuários, obtidos sob confidencialidade de uma empresa provedora de serviços 


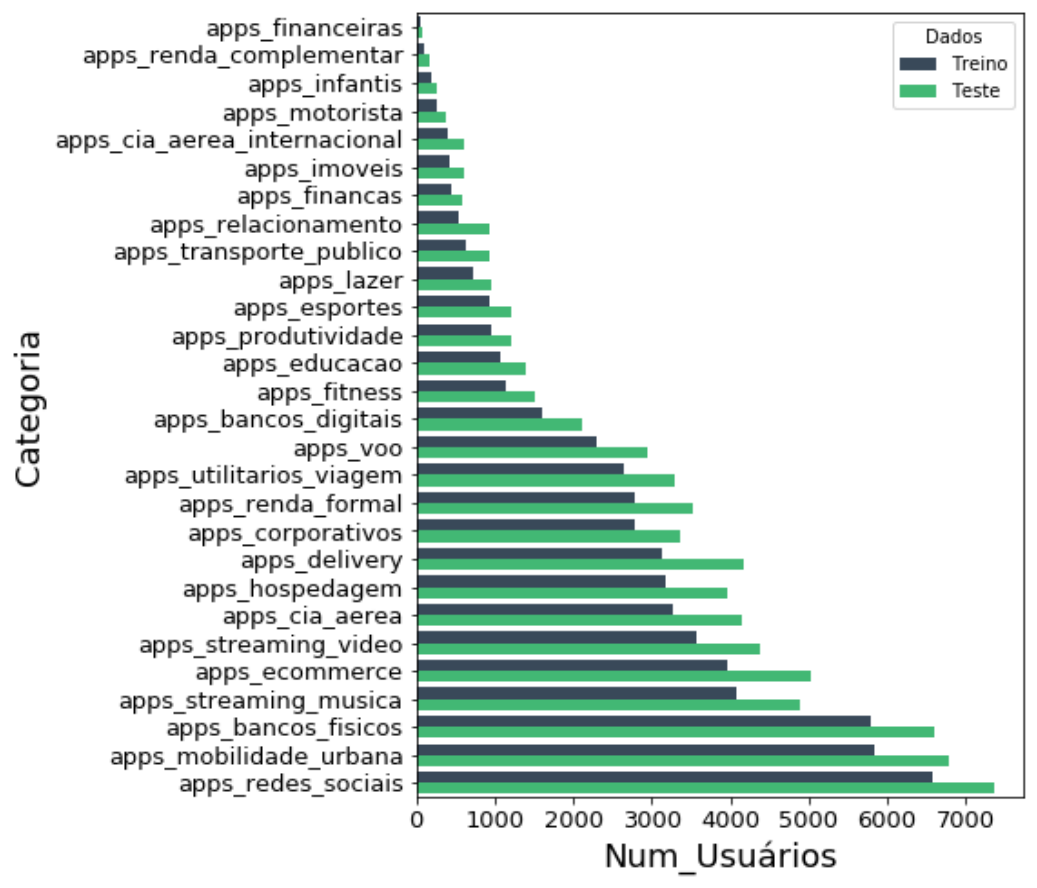

Figura 1. Número de usuários por categoria nas fases de treino e teste.

móveis. Estes dados foram coletados do dia 04 de Junho de 2019 ao dia 08 de Agosto de 2019. Para que os resultados fiquem coerentes, todos os 7.406 usuários participaram da coleta em todo o período. Ou seja, não entrou nenhum usuário novo e nenhum usuário abandonou a coleta no meio do período. Durante esse período, a cada dia um registro é gerado por usuário, indicando quais, dentre uma lista de 249 aplicativos, o usuário possui instalado. O conjunto de dados possui 249 aplicativos diferentes (Uber, Facebook, Nubank, Mercado Livre, Spotify, por exemplo) que podem ser agrupados em 28 categorias (Bancos Digitais, Esportes, E-Commerce, dentre outros).

Neste trabalho, o primeiro dia será utilizado para o treinamento, e os demais dias para testes dos modelos de recomendação. Em outras palavras, os dados representando os aplicativos existentes nos dispositivos dos usuários no dia 04 de Junho são usados para criar o modelo de recomendação. Em seguida, o modelo é aplicado e testado com os dados dos dias seguintes, para validar os modelos com base nos aplicativos que foram instalados. Na Figura 1, é possível ver a quantidade de usuários que cada categoria possui no dia de treino e nos dias de teste. É possível ver que o número de usuários aumentou em todas as categorias durante o período avaliado, ou seja, os usuários instalaram algum aplicativo da categoria. Com uma solução de recomendação, espera-se que uma parcela desses usuários que instalaram os aplicativos seja detectada antes da instalação.

Além dos aplicativos existentes no dispositivo móvel, também foi fornecida uma localização aproximada (por motivos de privacidade) da residência dos usuários. Para realização deste estudo, os dados foram enriquecidos com as informações dos setores censitários coletados da base do IBGE (Instituto Brasileiro de Geografia e Estatística), referentes ao local de residência dos usuários. A base de dados demográficos foi formatada, preparada e organizada para que métricas relevantes fossem extraídas. Ao todo, são 24 novas informações, que foram selecionadas por estarem potencialmente associadas com 
a decisão de se instalar um aplicativo ou não. Dentre essas características, tem-se por exemplo, a média de moradores por domicílio, média do rendimento por morador, taxa de alfabetizados e taxa de moradores em cada faixa etária e raça.

\section{ANCESTOR: Modelo de Recomendação baseado em Dados Demográficos}

Esta Seção descreve os detalhes da solução proposta, e os resultados da comparação com uma solução base. A solução base, denominada ALBERTA, é uma solução tradicional de filtro colaborativo para esse problema, e utiliza como itens apenas os aplicativos que os usuários têm instalado. Já o modelo proposto, chamado ANCESTOR, utiliza, além dos aplicativos, dados demográficos do IBGE como itens. Dessa forma, a hipótese de que os dados demográficos representam informações relevantes para a previsão de instalação de aplicativos pode ser validada ou refutada.

\subsection{Detalhes da Solução}

Filtros colaborativos funcionam construindo uma base de dados com as preferências de itens por usuários. Um novo usuário é comparado com essa base e são encontrados seus vizinhos, que são outros usuários que têm interesses similares. As recomendações são então feitas com base nos seus vizinhos. Existem dois tipos de abordagens possíveis para filtros colaborativos: Usuário-Item e Item-Item.

A abordagem Usuário-Item utiliza uma matriz de Usuários $\times$ Itens como base de dados para encontrar os vizinhos. Entretanto, tal método não apresenta boa escalabilidade em relação ao crescimento de usuários, principalmente caso haja interesse em conhecer alguns milhares de vizinhos de algum usuário a fim de fazer recomendações mais precisas [Sarwar et al. 2001].

Outra forma é a Item-Item, em que a similaridade é primeiramente observada no que se refere aos itens, criando uma matriz simétrica de Itens $\times$ Itens como base de dados. Como o relacionamento entre itens é relativamente estático, filtros colaborativos Item-Item podem ser capazes de prover a mesma qualidade da abordagem Usuário-Item com menor custo computacional e melhor escalabilidade. Por exemplo, é menos relevante se um usuário $U_{1}$ possui outros usuários similares a ele, do que se muitos usuários que possuem o aplicativo $A_{1}$ instalado também possuem um outro aplicativo $A_{2}$ e o usuário $U_{1}$ possui $A_{1}$. Nesse caso, a recomendação de $A_{2}$ para o usuário $U_{1}$ pode ser uma boa alternativa.

Neste trabalho, foi utilizada a abordagem Item-Item pois seu tempo de execução não cresce assintoticamente com o aumento da quantidade de usuários e, no contexto do problema de recomendação de aplicativos, é esperado que se tenha um grande número de usuários, que é significativamente maior que o número de aplicativos.

$\mathrm{Na}$ abordagem Item-Item, um item deve indicar alguma característica do usuário. Para a solução base ALBERTA, cada item representa um aplicativo, e o seu valor é 1 (um) caso o usuário tenha o aplicativo instalado, e 0 (zero) caso contrário. Para a proposta ANCESTOR, além desses itens representando cada um dos 239 aplicativos considerados no trabalho, são acrescentados outros 24 itens referentes às características demográficas do local de residência do usuário, normalizadas entre 0 e 1 . Dentre esses 24 itens, temos por exemplo o percentual de moradores da região na faixa etária entre 0 e 10 anos (e 
também em todas as outras faixas etárias), a renda per capita dos moradores da região, a taxa de alfabetização da região, e a distribuição de raça dos moradores.

\subsection{Resultados e Discussão}

Para avaliar a qualidade da proposta, foram aplicadas as métricas de precisão (porcentagem do total de acertos em relação ao total de instalações previstas para cada aplicativo), revocação (porcentagem do total de acertos em relação ao total de instalações que realmente ocorreram de cada aplicativo) e F-Score (balanceamento através da média harmônica entre precisão e revocação).

Além dessas três métricas, foi calculada também uma métrica importante que é o Lift, que indica a taxa de melhoria em relação a amostras aleatórias. Ou seja, é comparada a precisão de uma amostra aleatória com a precisão do modelo. O valor do Lift indica quantas vezes o modelo foi melhor (ou pior, caso o valor seja negativo) que a estratégia de selecionar usuários aleatórios. Para o cálculo do Lift médio de um aplicativo, foi utilizada a precisão média de 30 amostras geradas aleatoriamente. Este processo foi repetido para diferentes tamanhos de amostra $(8 \%, 9 \%, 10 \%, 12 \%, 15 \%, 20 \%, 30 \%, 40 \%, 50 \%$ e $60 \%$ do total de usuários), buscando assim averiguar o comportamento geral dos modelos.

De acordo com as métricas utilizadas, os valores são calculados para cada aplicativo individualmente. A Figura 2 mostra a distribuição da precisão, revocação e f-score considerando todos os aplicativos. Como pode-se ver, o ANCESTOR, no geral, se sobressai ao modelo ALBERTA. Isso mostra que a adição das informações demográficas do IBGE contribuíram para um maior acerto da predição das instalações da maioria dos aplicativos, tanto do ponto de vista de acerto dos aplicativos recomendados, quanto do ponto de vista dos aplicativos que realmente foram instalados. Inclusive, observamos que a precisão máxima alcançada pelo modelo ANCESTOR é quase o dobro da alcançada pelo ALBERTA. Mesmo que este comportamento se dê apenas para alguns aplicativos e não represente a maioria, a vantagem obtida para estes pode ser bem relevante quando pensamos em grandes quantidades de usuários.
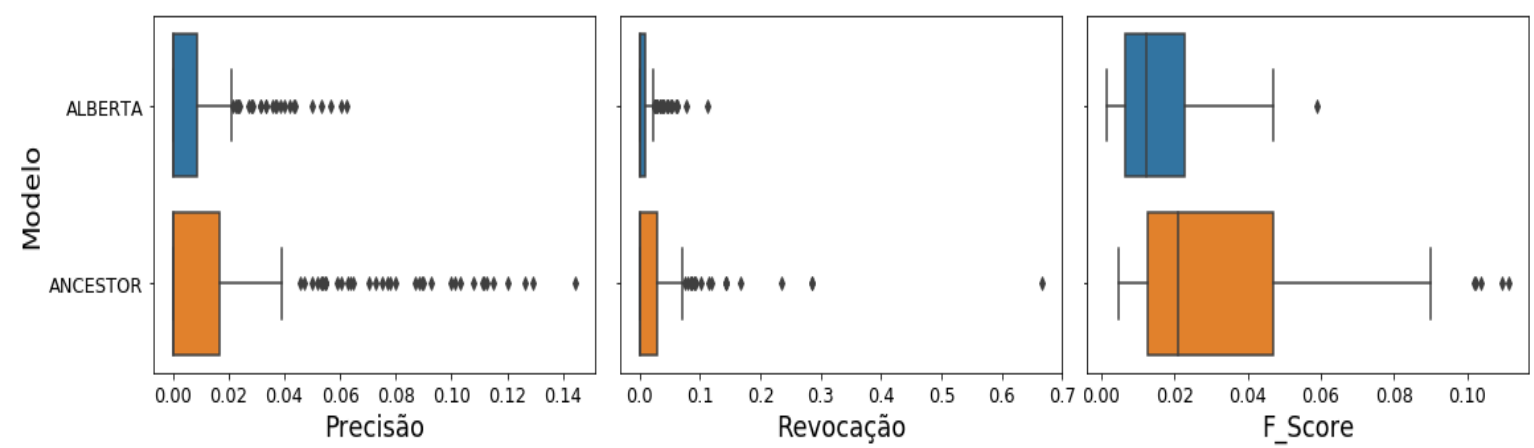

Figura 2. Precisão, Revocação e F-Score dos aplicativos.

Apesar de a precisão ser baixa em se tratando de modelos preditivos, algumas observações são importantes para o contexto de recomendação de instalação de aplicativos. Primeiramente, pode-se perceber que ao se acrescentar as informações demográficas dos usuários, a precisão aumentou para a maioria dos aplicativos. Isso mostra que a similaridade dos usuários em termos das características demográficas é um fator relevante. 

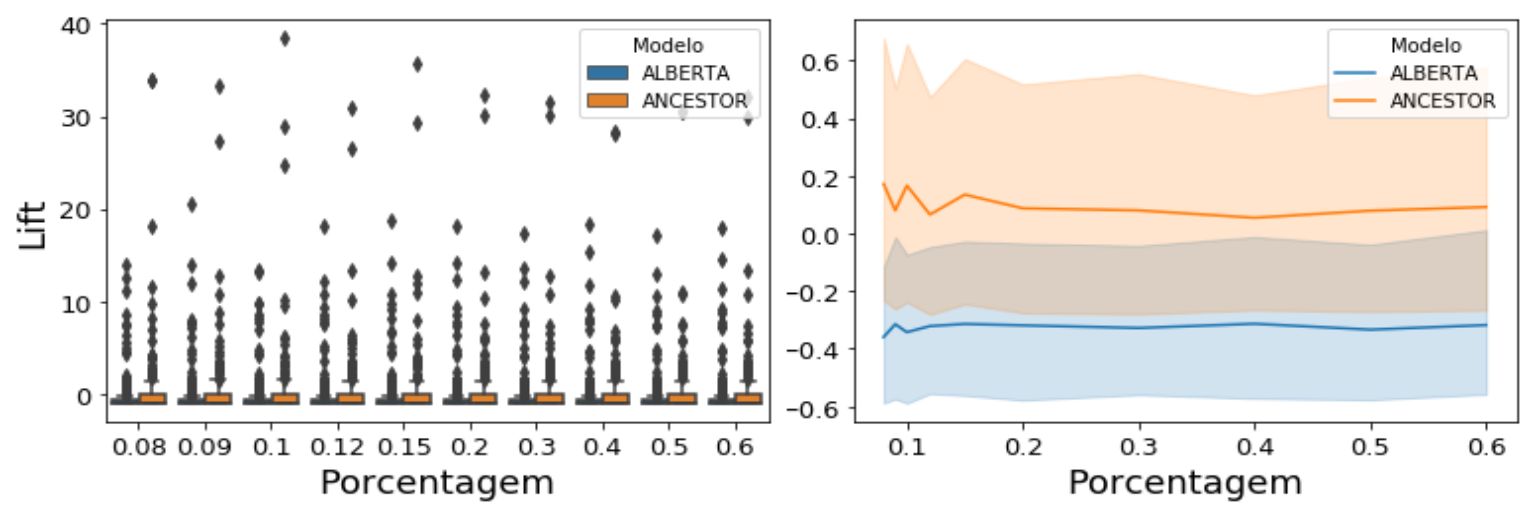

Figura 3. a) Lift alcançado para cada tamanho de amostra. b) Curva do Lift médio para todos os tamanhos de amostra, com intervalo de confiança de $95 \%$.

Outra consideração diz respeito à taxa de conversão ou retorno quando uma campanha é feita para atrair novos usuários para determinado aplicativo. Pode ser que, mesmo com uma precisão baixa, a taxa de aumento (i.e., Lift) ao ter como alvo usuários recomendados pelo modelo seja maior do que ao se utilizar amostras aleatórias de usuários. Ou seja, o retorno do investimento por usuário alcançado será maior. O resultado de tal análise pode ser visto na Figura 3 (a). Nela é possível perceber que o Lift não possui grande variância para as mudanças de tamanho da amostra, além de que o ANCESTOR possui, no geral, os melhores resultados. Além disso, na Figura 3 (b), vemos que mesmo com o intervalo de confiança, o ANCESTOR sempre possui uma taxa de levantamento maior que o ALBERTA.

Esses resultados mostram que, em geral, ao se utilizar o modelo de recomendação ANCESTOR, a taxa de retorno tende a ser mais efetiva do que ao se utilizar amostras aleatórias de usuários como alvo. Ou seja, a chance de um usuário recomendado pelo modelo ANCESTOR instalar determinado aplicativo após uma campanha de marketing direcionada a ele é maior do que se a campanha fosse direcionada a usuários escolhidos aleatoriamente.

Para melhor visualizar o comportamento dos modelos em relação aos aplicativos, calculamos a precisão média para cada categoria de aplicativo. Com isso, esperamos poder identificar o motivo de a precisão de alguns aplicativos aumentarem relativamente com a adição de dados demográficos. Na Figura 4, pode ser observado que a maior parte das categorias com maior precisão média dependem de fatores demográficos, como por exemplo aplicativos de mobilidade urbana e delivery, que estão mais presentes em cidades ou locais de alto índice populacional. Já aplicativos referentes a companhias aéreas, streaming de vídeo ou música, hospedagem e e-commerce dependem de outros fatores, como a renda, por exemplo. Outras categorias de aplicativos, como aplicativos infantis e de relacionamento, não dependem tanto dos fatores demográficos do local e, portanto, o ANCESTOR não apresenta uma melhora significativa em relação ao ALBERTA.

Para apresentar mais detalhes, a Tabela 2 mostra os 20 aplicativos com melhor precisão do modelo ALBERTA. Pode-se perceber que a maior parte dos melhores resultados são referentes a aplicativos que obtiveram relativamente poucas instalações ou são concorrentes diretos de outros aplicativos considerados os principais de seus segmentos, como por exemplo James, Uber Eats e Rappi (concorrentes do iFood), e Happn (concor- 


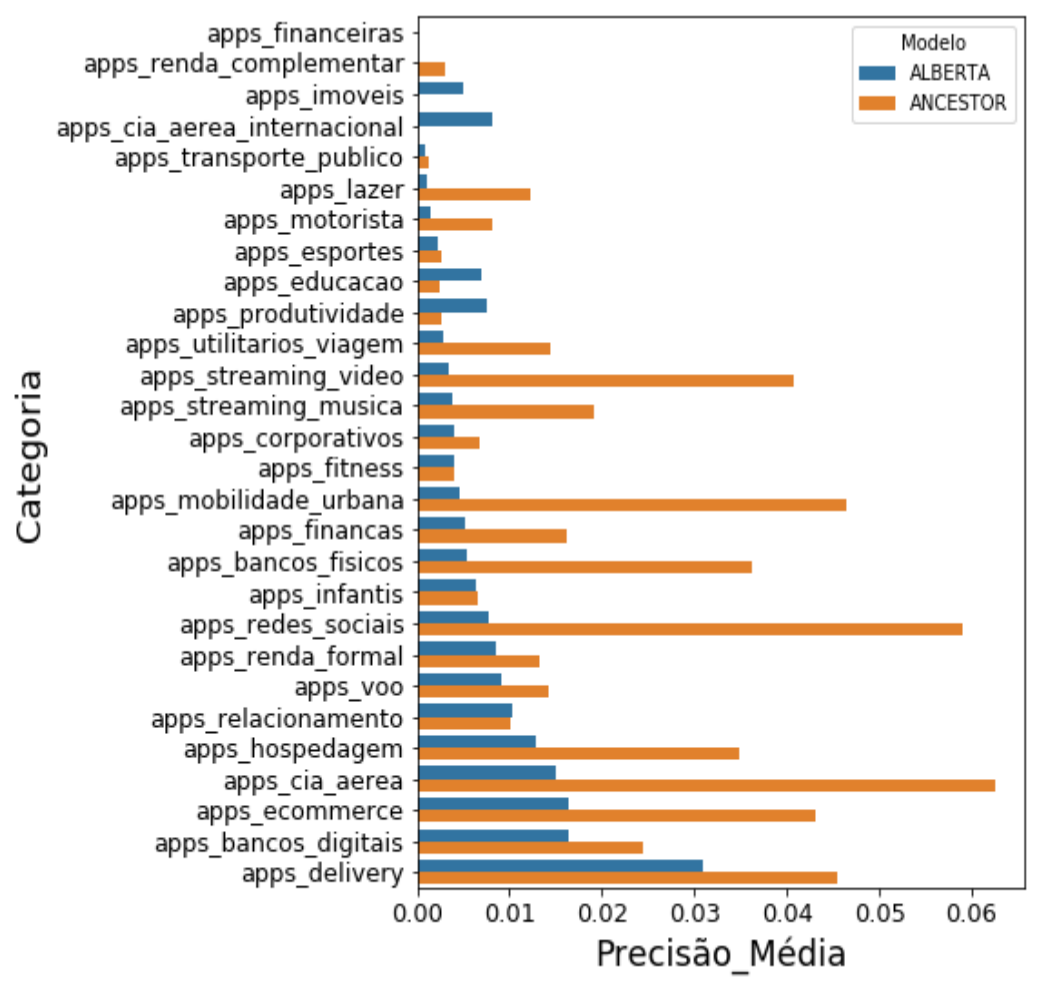

Figura 4. Precisão Média por Categoria.

rente do Tinder).

Por outro lado, mesmo entre os aplicativos com melhor precisão para o ALBERTA, alguns (como AliExpress, Alelo, Uber Eats e Netshoes), possuem uma precisão menor do que ANCESTOR. Dentre os piores desempenhos do ANCESTOR, estão os aplicativos de companhias aéreas internacionais. Isso pode ser explicado pelo fato de que não haviam muitos dados acerca deste tipo de aplicativo, sendo somente 413 usuários no período utilizado para treino, em comparação aos 3281 usuários que possuíam aplicativos de companhias aéreas nacionais no mesmo período. Esta explicação casa com o fato de que não há aplicativos de companhias aéreas nacionais dentre os melhores para o modelo ALBERTA.

Diferentemente do ALBERTA, o ANCESTOR se sai melhor dentre os aplicativos que possuem alto índice de instalação, chegando a 14,45\% de precisão, como vemos na Tabela 3. Também pode-se perceber que a menor precisão indicada nesta tabela ainda é maior que a maior alcançada pelo ALBERTA. Vale também ressaltar que são mais frequentes os aplicativos pioneiros em suas áreas, como Uber, iFood e Netflix, além dos aplicativos de redes sociais. Outro ponto de destaque é o Lift alcançado pelo ANCESTOR, uma vez que todos os aplicativos obtêm melhora, ou se mantêm, no alcance de usuários em relação a amostras aleatórias. Considerando também o Lift, vemos que dentre os aplicativos que aparecem nesta tabela, alguns foram instalados por poucos usuários, levando a uma taxa de levantamento bem alta nestes casos, chegando a ser quase 31 vezes melhor que as amostras aleatórias. 
Tabela 2. Tabela das estatísticas dos 20 aplicativos que obtiveram melhor precisão para o modelo ALBERTA.

\begin{tabular}{|c|c|c|c|c|c|c|c|}
\hline Aplicativo & $\begin{array}{c}\text { Total } \\
\text { Instalações }\end{array}$ & $\begin{array}{c}\text { Precisão } \\
\text { ALBERTA }\end{array}$ & $\begin{array}{c}\text { Precisão } \\
\text { ANCESTOR }\end{array}$ & $\begin{array}{l}\text { Revocação } \\
\text { ALBERTA }\end{array}$ & $\begin{array}{c}\text { Revocação } \\
\text { ANCESTOR }\end{array}$ & $\begin{array}{c}\text { Lift } \\
\text { ALBERTA }\end{array}$ & $\begin{array}{c}\text { Lift } \\
\text { ANCESTOR }\end{array}$ \\
\hline Udemy & 54 & $6,25 \%$ & $0,00 \%$ & $3,70 \%$ & $0,00 \%$ & 7,65 & $-1,00$ \\
\hline James & 52 & $6,06 \%$ & $0,00 \%$ & $3,85 \%$ & $0,00 \%$ & 7,48 & $-1,00$ \\
\hline Happn & 131 & $5,67 \%$ & $1,41 \%$ & $6,11 \%$ & $1,53 \%$ & 2,21 & $-0,20$ \\
\hline AliExpress & 449 & $5,31 \%$ & $7,05 \%$ & $2,90 \%$ & $3,56 \%$ & $-0,13$ & 0,16 \\
\hline Uber Eats & 759 & $5,00 \%$ & $6,45 \%$ & $1,45 \%$ & $1,05 \%$ & $-0,51$ & $-0,37$ \\
\hline KayaK & 150 & $4,40 \%$ & $0,00 \%$ & $2,67 \%$ & $0,00 \%$ & 1,14 & $-1,00$ \\
\hline Next & 65 & $4,35 \%$ & $0,99 \%$ & $4,62 \%$ & $1,54 \%$ & 3,91 & 0,13 \\
\hline FGTS & 332 & $4,17 \%$ & $3,05 \%$ & $2,11 \%$ & $1,51 \%$ & $-0,07$ & $-0,32$ \\
\hline KLM & 20 & $4,00 \%$ & $0,00 \%$ & $0.05 \%$ & $0,00 \%$ & 14,71 & $-1,00$ \\
\hline Slack & 23 & $3,85 \%$ & $0,00 \%$ & $4,35 \%$ & $0,00 \%$ & 11,18 & $-1,00$ \\
\hline Rappi & 420 & $3,70 \%$ & $5,00 \%$ & $1,43 \%$ & $0,71 \%$ & $-0,34$ & $-0,12$ \\
\hline Netshoes & 207 & $3,69 \%$ & $5,46 \%$ & $3,86 \%$ & $6,28 \%$ & 0,34 & 0,98 \\
\hline Air France & 27 & $3,57 \%$ & $0,00 \%$ & $3,70 \%$ & $0,00 \%$ & 9,45 & $-1,00$ \\
\hline Centauro & 95 & $3,36 \%$ & $0,46 \%$ & $5,26 \%$ & $1,05 \%$ & 1,67 & $-0,65$ \\
\hline DAZN & 100 & $3,33 \%$ & $1,78 \%$ & $4,00 \%$ & $3,00 \%$ & 1,46 & 0,32 \\
\hline Wish & 443 & $3,16 \%$ & $5,38 \%$ & $1,81 \%$ & $4,51 \%$ & $-0,47$ & $-0,11$ \\
\hline Google Analytics & 13 & $3,12 \%$ & $0,00 \%$ & $7,69 \%$ & $0,00 \%$ & 17,68 & $-1,00$ \\
\hline Copa Airlines & 37 & $2,86 \%$ & $0,00 \%$ & $5,41 \%$ & $0,00 \%$ & 4,76 & $-1,00$ \\
\hline Trivago & 235 & $2,82 \%$ & $1,96 \%$ & $2,55 \%$ & $0,85 \%$ & $-0,11$ & $-0,39$ \\
\hline Alelo & 325 & $2,80 \%$ & $6,28 \%$ & $2,15 \%$ & $4,62 \%$ & $-0,36$ & 0,43 \\
\hline
\end{tabular}

Tabela 3. Tabela das estatísticas dos 20 aplicativos que obtiveram melhor precisão para o modelo ANCESTOR.

\begin{tabular}{|l|r|r|r|r|r|r|r|}
\hline \multicolumn{1}{|c|}{ Aplicativo } & $\begin{array}{c}\text { Total } \\
\text { Instalações }\end{array}$ & $\begin{array}{c}\text { Precisão } \\
\text { ALBERTA }\end{array}$ & $\begin{array}{c}\text { Precisão } \\
\text { ANCESTOR }\end{array}$ & $\begin{array}{c}\text { Revocação } \\
\text { ALBERTA }\end{array}$ & $\begin{array}{c}\text { Revocação } \\
\text { ANCESTOR }\end{array}$ & $\begin{array}{c}\text { Lift } \\
\text { ALBERTA }\end{array}$ & $\begin{array}{c}\text { Lift } \\
\text { ANCESTOR }\end{array}$ \\
\hline Uber & 961 & $0,23 \%$ & $14,45 \%$ & $0,10 \%$ & $9,05 \%$ & -0.98 & 0,11 \\
\hline Messenger & 937 & $2,12 \%$ & $12,92 \%$ & $1,07 \%$ & $8,64 \%$ & $-0,83$ & 0,02 \\
\hline Instagram & 896 & $0,69 \%$ & $12,64 \%$ & $0,33 \%$ & $8,59 \%$ & $-0,94$ & 0,05 \\
\hline Whatsapp & 894 & $0,22 \%$ & $12,02 \%$ & $0,11 \%$ & $10,07 \%$ & $-0,98$ & 0,01 \\
\hline Facebook & 855 & $0,63 \%$ & $11,50 \%$ & $0,35 \%$ & $9,12 \%$ & $-0,95$ & 0,00 \\
\hline iFood & 889 & $0,69 \%$ & $11,24 \%$ & $0,22 \%$ & $3,15 \%$ & $-0,94$ & $-0,06$ \\
\hline OLX & 651 & $1,65 \%$ & $11,16 \%$ & $0,92 \%$ & $7,53 \%$ & $-0,81$ & 0,27 \\
\hline Guiabolso & 70 & $1,89 \%$ & $11,11 \%$ & $1,43 \%$ & $5,71 \%$ & 1,02 & 10,83 \\
\hline MercadoLivre & 692 & $1,33 \%$ & $10,79 \%$ & $0,58 \%$ & $5,92 \%$ & $-0,86$ & 0,16 \\
\hline Netflix & 780 & $0,62 \%$ & $10,33 \%$ & $0,26 \%$ & $5,26 \%$ & $-0,94$ & $-0,02$ \\
\hline 99 Taxis & 767 & $0,00 \%$ & $10,10 \%$ & $0,00 \%$ & $3,91 \%$ & $-1,00$ & $-0,03$ \\
\hline Steam & 24 & $0,00 \%$ & $10,00 \%$ & $0,00 \%$ & $4,17 \%$ & $-1,00$ & 30,09 \\
\hline Booking & 597 & $1,12 \%$ & $9,28 \%$ & $0,67 \%$ & $3,69 \%$ & $-0,86$ & 0,15 \\
\hline LinkedIn & 523 & $0,84 \%$ & $8,96 \%$ & $0,38 \%$ & $3,63 \%$ & $-0,88$ & 0,27 \\
\hline Spotify & 686 & $0,62 \%$ & $8,95 \%$ & $0,29 \%$ & $4,23 \%$ & $-0,93$ & $-0,03$ \\
\hline LATAM & 479 & $1,58 \%$ & $8,85 \%$ & $1,04 \%$ & $3,55 \%$ & $-0,76$ & 0,36 \\
\hline Prime Video & 96 & $1,75 \%$ & $8,70 \%$ & $1,04 \%$ & $2,08 \%$ & 0,39 & 5,70 \\
\hline GOL & 776 & $1,55 \%$ & $7,97 \%$ & $0,64 \%$ & $2,84 \%$ & $-0,85$ & $-0,24$ \\
\hline Pinterest & 359 & $0,71 \%$ & $7,79 \%$ & $0,56 \%$ & $6,69 \%$ & $-0,85$ & 0,61 \\
\hline Twitch & 18 & $0,00 \%$ & $7,69 \%$ & $0,00 \%$ & $5,56 \%$ & $-1,00$ & 30,73 \\
\hline
\end{tabular}

\section{Conclusões e Trabalhos Futuros}

Este trabalho apresentou uma solução para recomendação de aplicativos a usuários com base nos seus aplicativos instalados e informações demográficas. Os resultados apresentados mostram que a solução proposta supera a solução de base em vários aspectos, atingindo bons resultados em termos de precisão, revocação e lift. Com isso, a hipótese de que dados demográficos são importantes para ajudar na recomendação de instalação de 
aplicativos é reforçada. Além disso, o fato de a solução não necessitar de dados históricos, tanto dos aplicativos quanto dos usuários, faz com que a construção do modelo seja mais simples.

Como trabalhos futuros, pretende-se tratar casos em que hajam poucas instalações de um determinado aplicativo nos dados de treino. Além disso, também é importante avaliar detalhadamente quais os principais fatores demográficos que estão associados à instalação de determinados aplicativos.

\section{Agradecimentos}

Agradecemos à CAPES, CNPq e Fapemig pelo suporte financeiro ao projeto.

\section{Referências}

Cheng, V. C., Chen, L., Cheung, W. K., and Fok, C.-k. (2018). A heterogeneous hidden markov model for mobile app recommendation. Knowledge and Information Systems, 57(1):207-228.

Frey, R. M., Xu, R., Ammendola, C., Moling, O., Giglio, G., and Ilic, A. (2017). Mobile recommendations based on interest prediction from consumer's installed apps-insights from a large-scale field study. Information Systems, 71:152 - 163.

Intelligence, G. (2019). The mobile economy 2019. online.

Liu, B., Kong, D., Cen, L., Gong, N. Z., Jin, H., and Xiong, H. (2015). Personalized mobile app recommendation: Reconciling app functionality and user privacy preference. In Proceedings of the Eighth ACM International Conference on Web Search and Data Mining, WSDM '15, pages 315-324, New York, NY, USA. ACM.

Liu, B., Wu, Y., Gong, N. Z., Wu, J., Xiong, H., and Ester, M. (2016). Structural analysis of user choices for mobile app recommendation. ACM Trans. Knowl. Discov. Data, 11(2):17:1-17:23.

Ma, Q., Muthukrishnan, S., and Simpson, W. (2016). App2vec: Vector modeling of mobile apps and applications. In 2016 IEEE/ACM International Conference on Advances in Social Networks Analysis and Mining (ASONAM), pages 599-606.

Pan, W., Aharony, N., and Pentland, A. S. (2011). Composite social network for predicting mobile apps installation. In Proceedings of the Twenty-Fifth AAAI Conference on Artificial Intelligence, AAAI'11, pages 821-827. AAAI Press.

Peng, M., Zeng, G., Sun, Z., Huang, J., Wang, H., and Tian, G. (2018). Personalized app recommendation based on app permissions. World Wide Web, 21(1):89-104.

Sarwar, B. M., Karypis, G., Konstan, J. A., Riedl, J., et al. (2001). Item-based collaborative filtering recommendation algorithms. $W w w, 1: 285-295$.

Xu, X., Dutta, K., Datta, A., and Ge, C. (2018). Identifying functional aspects from user reviews for functionality-based mobile app recommendation. Journal of the Association for Information Science and Technology, 69(2):242-255.

Yin, H., Chen, L., Wang, W., Du, X., Nguyen, Q. V. H., and Zhou, X. (2017). Mobi-sage: A sparse additive generative model for mobile app recommendation. In 2017 IEEE 33rd International Conference on Data Engineering (ICDE), pages 75-78. 